\title{
Primary vaginal malignancies: a single oncology centre experience
}

\section{Mariana Lima, Gisela Rio, Mariana Horta \& Teresa Margarida Cunha}

To cite this article: Mariana Lima, Gisela Rio, Mariana Horta \& Teresa Margarida Cunha (2019): Primary vaginal malignancies: a single oncology centre experience, Journal of Obstetrics and Gynaecology, DOI: 10.1080/01443615.2019.1579786

To link to this article: https://doi.org/10.1080/01443615.2019.1579786

\section{Published online: 25 Apr 2019.}

Submit your article to this journal

Џll Article views: 7

View Crossmark data ¿ 


\title{
Primary vaginal malignancies: a single oncology centre experience
}

\author{
Mariana Lima ${ }^{a}$, Gisela Rio $^{b}$, Mariana Horta $^{c}$ and Teresa Margarida Cunha ${ }^{c}$ \\ a Department of Radiology, Centro Hospitalar de Lisboa Central (Hospital de Santo António dos Capuchos), Alameda de Santo António dos

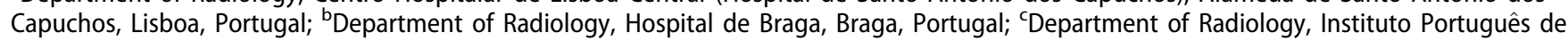 \\ Oncologia de Lisboa Francisco Gentil, Rua Prof. Lima Basto, Lisboa, Portugal
}

\section{ABSTRACT}

Primary vaginal malignancies constitute a rare entity. The aim of this study was to review all primary vaginal malignancies diagnosed in an oncologic referral centre over 11 years. A total of 35 cases were retrospectively analysed, including clinical and MRI features. Squamous cell carcinoma (SCC) was the most frequent histologic subtype (77.1\%), followed by adenocarcinoma (14.3\%). There was no statistically significant difference for the mean age at diagnosis or for the mean largest diameter of the tumour. Most SCCs (95\%) were homogeneous on T2-weighted imaging, while all adenocarcinomas were heterogeneous $(p=.0001)$. Concerning location, both SCCs $(59.3 \%)$ and adenocarcinomas $(80 \%)$ occurred more often on the upper third. However, regarding the wall of origin, all adenocarcinomas originated on the anterior vaginal wall $(p=.0002)$, while SCCs $(62.5 \%)$ had a predisposition for the posterior wall $(p=.017)$. Regarding the history of previous hysterectomy, in the SCC group, $73.3 \%$ of patients with previous hysterectomies had cervical dysplasia $(p=.018)$.

\section{IMPACT STATEMENT}

- What is already known on this subject? MRI plays an important role in the initial approach of primary vaginal malignancies. In previous studies, it is said that SCCs usually appear homogeneous on T2WI, with the intermediate-high signal, while adenocarcinomas are often homogeneously hyperintense. Regarding location, it is known that SCCs usually arise from the posterior wall of the upper third, while adenocarcinomas often originate on the anterior wall of the proximal third.

- What do the results of this study add? In this study, we found that all of our cases of adenocarcinomas were heterogeneous on $\mathrm{T} 2 \mathrm{WI}$, with high-signal intensity areas, while SCCs were predominantly homogeneous, and this association was statistically significant. We could also confirm the data in the literature regarding the most common location of these tumours. This study also showed an association between vaginal SCC and a previous hysterectomy with cervical dysplasia.

- What are the implications of these findings for clinical practice and/or further research? The histologic type of vaginal malignancy has clinical and management impact. Although MRI is usually performed after histologic characterisation, this is not always the case. We think that this study can constitute a starting point to better understand the MRI features of these rare tumours. Although this technique will obviously never preclude histologic characterisation, it may provide some initial hint on the type of tumour and its aggressiveness.

\section{KEYWORDS}

Primary vaginal

malignancies; vaginal adenocarcinoma; vaginal squamous cell carcinoma; MRI features; T2-weighted imaging

\section{Introduction}

Primary vaginal malignancies are rare, corresponding to approximately $1-2 \%$ of gynaecologic malignancies (Parikh et al. 2008). In fact, vaginal cancer is more commonly secondary (Chang et al. 1988) and $80-90 \%$ of vaginal tumours result from direct extension of primary bladder, vulvar, cervical or rectal tumours. Also, the vagina may present distant metastases (López et al. 2005).

The diagnosis of primary vaginal carcinoma can only be established when a tumour arises exclusively from the vagina, with no involvement of the vulva or the external os. Also, other gynaecologic malignancies must be excluded (Parikh et al. 2008). The incidence of primary vaginal cancer increases with age (Taylor et al. 2007; Gardner et al. 2015). It usually presents with painless vaginal bleeding/discharge, urinary symptoms, pelvic pain and foreign body sensation (Grant et al. 2010).

About $85-87 \%$ of primary tumours are squamous cell carcinomas (SCCs). The second most common histologic type is adenocarcinoma ( $9 \%$ of primary vaginal malignancies) (Parikh et al. 2008). Less frequent malignancies include melanoma (3\%) and sarcoma (1\%) (Walker et al. 2011).

The vagina can be anatomically divided into thirds, which is important for classifying tumour location and relates with its lymphatic drainage. The lower third is the portion below the level of the bladder base, posteriorly to the urethra; the 
middle third locates at the level of the bladder base and the upper third corresponds to the level of the vaginal fornices (Gardner et al. 2015; Tsili 2019).

As for other gynaecologic malignancies, the staging of vaginal carcinoma is based on the International Federation of Gynaecology and Obstetrics (FIGO) staging system (Parikh et al. 2008; Gardner et al. 2015; Tsili 2019). Pelvic examination is still the primary modality for evaluating the extent of disease, as for cervical cancer. However, it has several limitations, like the impossibility to detect metastatic lymph nodes and difficulty for depicting tumour infiltration (Gardner et al. 2015; Tsili 2019). Because of that, FIGO 2009 recommends the use of cross-sectional imaging (CT/MRI) (Tsili 2019). MRI provides a better evaluation of tumour volume and local extension, like parametrial and pelvic sidewall involvement, as well as spread to the bladder, urethra, rectum and lymph nodes (Parikh et al. 2008; Tsili 2019). MRI is also very useful for depicting pelvic anatomy before surgery and for radiation therapy planning (Parikh et al. 2008; Tsili 2019).

The histologic characterisation of vaginal malignancies is frequently established prior to MRI examination since the procedure is often easy to perform. However, this is not true for all vaginal tumours, particularly the nonsquamous ones. In fact, MRI examination may be performed before the histologic characterisation and can even play a role in determining a route for biopsy (Parikh et al. 2008).

However, the previous studies on this data are limited since this is a rare disease with a small case series. Hence, the aim of this study was to retrospectively review all the cases of this rare entity that were diagnosed in our oncologic centre from 2006 to 2017, including clinical and MRI characteristics. We aimed to compare our results with the literature data and look for possible distinctive features between SCC and adenocarcinoma. It was also our aim to study the incidence of previous hysterectomy and its possible association with the development of primary vaginal malignancies.

\section{Materials and methods}

\section{Patients}

All patients diagnosed with primary vaginal cancer between February 2006 and September 2017 were included (35 patients).

\section{Data collection}

A retrospective analysis of the clinical charts was conducted. The following information was collected for each patient: age at diagnosis, histologic type, FIGO staging, history of previous hysterectomy and the reason for this procedure.

Retrospective MRI reading was performed by two radiologists, one of them with 20 years of experience in gynaecologic radiology. Some data were registered for all patients: T2WI (weighted-imaging) signal of the tumour, T1WI signal of the tumour, location of the tumour largest diameter and staging (FIGO 2009 staging criteria).

Two of the cases of SCC corresponded to VAIN (Vaginal intraepithelial neoplasia) III and, as such, were not visible on
MRI. For four other patients, the MRI was not available for review.

\section{MRI technique}

MR imaging was performed both with 1.5 and $3 T$ units. All $M R I$ examinations included the following sequences: axial FSE T1Wl; sagittal FSE T2-weighted imaging (T2WI) and axial oblique FSE T2WI (perpendicular to vagina). The examinations were performed after filling the vagina with ultrasound gel.

\section{Statistical analysis}

The statistical analysis was performed using IBM SPSS Statistics 23.0 (Chicago, IL). A descriptive analysis was conducted. Association between the different categorical variables and the histologic type (SCC/adenocarcinoma) was evaluated using Fisher's exact test. The continuous variables were studied with the $t$-test for the independent samples. A $p$-value less than .05 was considered statistically significant.

\section{Research ethics standards compliance}

All of the procedures performed in the studies involving human participants were in accordance with the ethical standards of the institutional research committee and with the 1964 Helsinki declaration and its later amendments. For this type of study, formal consent is not required.

\section{Results}

SCC was the most frequent histological subtype and accounted for 27 cases (77.1\%), followed by adenocarcinoma that corresponded to 5 cases (14.3\%). Glassy cell carcinoma, melanoma and botryoid sarcoma occurred as isolated cases.

The age range of the study population was 27-91 years (mean 62 years \pm 14 ). Regarding the mean age at diagnosis, we found no statistically significant difference $(p=.550)$ between SCC (63 years old) and adenocarcinoma ( 58 years old). The mean largest diameter of the tumour was $41.5 \mathrm{~mm}$ for SCC and $41.3 \mathrm{~mm}$ for adenocarcinoma, and there was also no statistically significant difference between them $(p=.998)$.

The MRI characteristics of the tumours are shown in Table 1. On MRI, SCC were predominantly homogeneous on T2-weighted imaging (95\%), while all adenocarcinomas were heterogeneous on T2-weighted imaging. With Fisher's exact test, this difference was statistically significant $(p=.0001)$. Their T2 behaviour is shown in Figures 1 and 2. There was only one case of heterogeneous SCC on T2-weighted imaging, which corresponded to a poorly differentiated SCC (Figure 3).

When analysing the T1 signal, we found that one case of adenocarcinoma showed hyperintense areas on T1 (Figure 4), while none of the cases of SCC had this behaviour. However, there was no statistically significant difference for this parameter $(p=.1923)$.

The location of the tumours is shown in Table 2. Both SCC (59.3\%) and adenocarcinomas (80\%) occurred predominantly 


\begin{tabular}{|c|c|c|c|c|c|c|}
\hline & & Squamous cell carcinoma & Adenocarcinoma & Glassy cell carcinoma & Melanoma & Botryoid sarcoma \\
\hline \multirow[t]{2}{*}{ T2 $\mathrm{Wl}^{\mathrm{a}}$ signal } & Homogeneous & $20(95.2 \%)$ & & $1(100 \%)$ & $1(100 \%)$ & $1(100 \%)$ \\
\hline & Heterogeneous & $1(4.8 \%)$ & $5(100 \%)$ & & & \\
\hline \multirow{2}{*}{ High-signal intensity areas on $\mathrm{T} 1 \mathrm{WI}^{\mathrm{a}}$} & Present & & $1(20 \%)$ & & & \\
\hline & Absent & $21(100 \%)$ & $4(80 \%)$ & $1(100 \%)$ & $1(100 \%)$ & $1(100 \%)$ \\
\hline Total & & 21 & 5 & 1 & 1 & 1 \\
\hline
\end{tabular}

Weighted-imaging (WI).

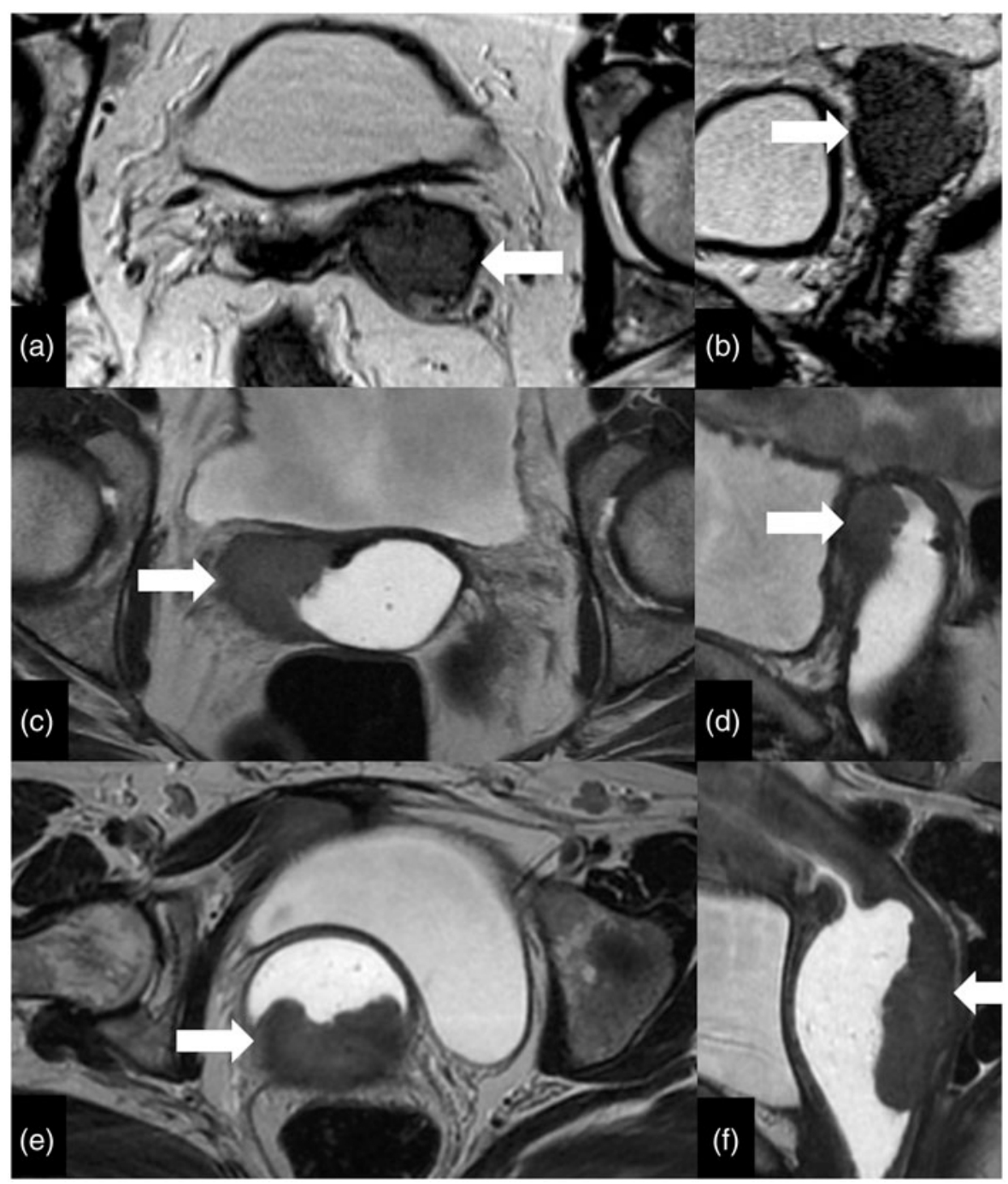

Figure 1. T2WI behaviour of squamous cell carcinomas. (a), (c) and (e) correspond to the axial T2-weighted images of three different squamous cell carcinomas and (b), (d) and (f) to their sagittal T2-weighted images. We can see that all the tumours show homogeneous intermediate signal on T2.

on the upper third of vagina, so there was no statistically significant difference between them for the vaginal third involved $(p=.626)$.

Most SCC originated on the posterior wall (62.5\%) and Fisher's exact test showed this association to be statistically significant $(p=.017)$. On the other hand, adenocarcinomas originated predominantly on the anterior wall $(80 \%)$, also with statistical significance $(p=.0002)$.

The FIGO staging for SCCs and adenocarcinomas is shown in Table 3. In the adenocarcinomas group, all of the cases were FIGO I or II and in the SCC group, $88.9 \%$ were FIGO I or II.

Table 4 shows data related to previous hysterectomies. We found no statistically significant difference between SCC and adenocarcinomas $(p=.625)$ since there was a high proportion of previous hysterectomies in both groups. However, when analysing the reason for that surgery, we found that in the SCC group there was an association with cervical dysplasia $(p=.018)$.

\section{Discussion}

The mean age of our patients was 62 years old, which approaches the 60 years old described in previous studies (Taylor et al. 2007). Our histologic types followed the data described in the literature (Parikh et al. 2008) since the vast majority of vaginal primary malignancies corresponded to 


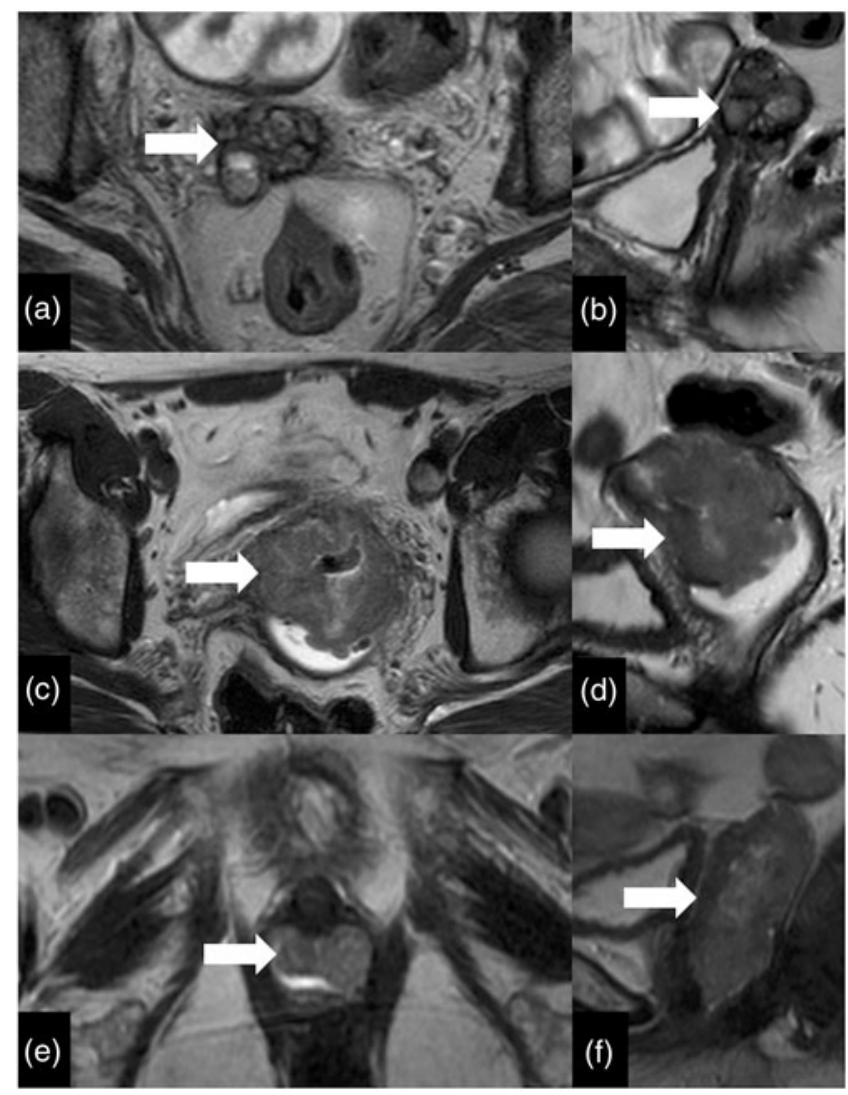

Figure 2. T2WI behaviour of adenocarcinomas. (a), (c) and (e) correspond to the axial T2-weighted images of three different adenocarcinomas and (b), (d) and ( $f$ ) to their sagittal T2-weighted images. All the tumours show heterogeneous signal intensity on $\mathrm{T} 2$, with foci of high signal intensity.

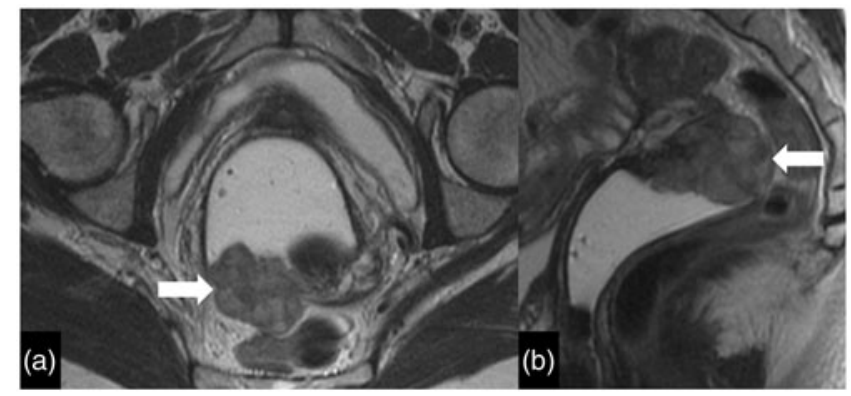

Figure 3. Poorly differentiated squamous cell carcinoma. Axial (a) and sagittal (b) T2-weighted images of a poorly differentiated squamous cell carcinoma that shows heterogeneous signal, with foci of high signal intensity on T2WI.
SCC. Some previous studies point out that adenocarcinomas tend to occur at a younger age, typically between the ages of 14 and 27 years (Parikh et al. 2008; Grant et al. 2010) because they can occur in foci of adenosis and endometriosis. However, in our study, we did not find a statistically significant difference in mean age at diagnosis between SCC and adenocarcinomas; the mean age at diagnosis for adenocarcinomas was 58 years. This can be related to our small sample size since there were only five adenocarcinomas. The mean largest diameter of the tumour was very similar in SCC $(41.5 \mathrm{~mm})$ and adenocarcinoma $(41.3 \mathrm{~mm})$. We also did not find any study in the literature showing a statistically significant difference between these.

Several previous studies have pointed out the typical T2 characteristics of adenocarcinomas and SCC. It has been described that SCCs show homogeneous intermediate to high signal intensity (Parikh et al. 2008; Grant et al. 2010), and adenocarcinomas tend to be homogeneously hyperintense on T2-weighted imaging (Parikh et al. 2008). However, in our study, we found that all five adenocarcinomas showed heterogeneous T2-signal because of the presence of regions with a higher T2-signal in the background of an intermediate to high signal intensity. Previous studies have already postulated that the intrinsic high T2-weighted signal intensity of adenocarcinomas represent mucin and may suggest the diagnosis (Grant et al. 2010). Some studies (Randall et al. 1986; Fetissof et al. 1990) have also pointed out that the presence of areas of high T2 signal intensity within a primary vaginal tumour should raise the possibility of it corresponding to a poorly differentiated SCC, an adenosquamous carcinoma or a mucinous adenocarcinoma. Another study also mentions that areas of high signal intensity on T2-weighted images can suggest mucin-producing adenocarcinoma (Siegelman et al. 1997). These data are in complete agreement with our results since all adenocarcinomas had this behaviour and the only SCC that was heterogeneous on T2-weighted imaging, with areas of high signal, was a poorly differentiated one.

Although it is stated in the literature that both SCC and adenocarcinomas are homogeneous and isointense to the muscles on T1-weighted imaging (Parikh et al. 2008; Grant et al. 2010), one of our adenocarcinomas showed foci of high signal intensity on $\mathrm{T} 1$. It is known that women with endometriosis are at risk of developing vaginal adenocarcinomas

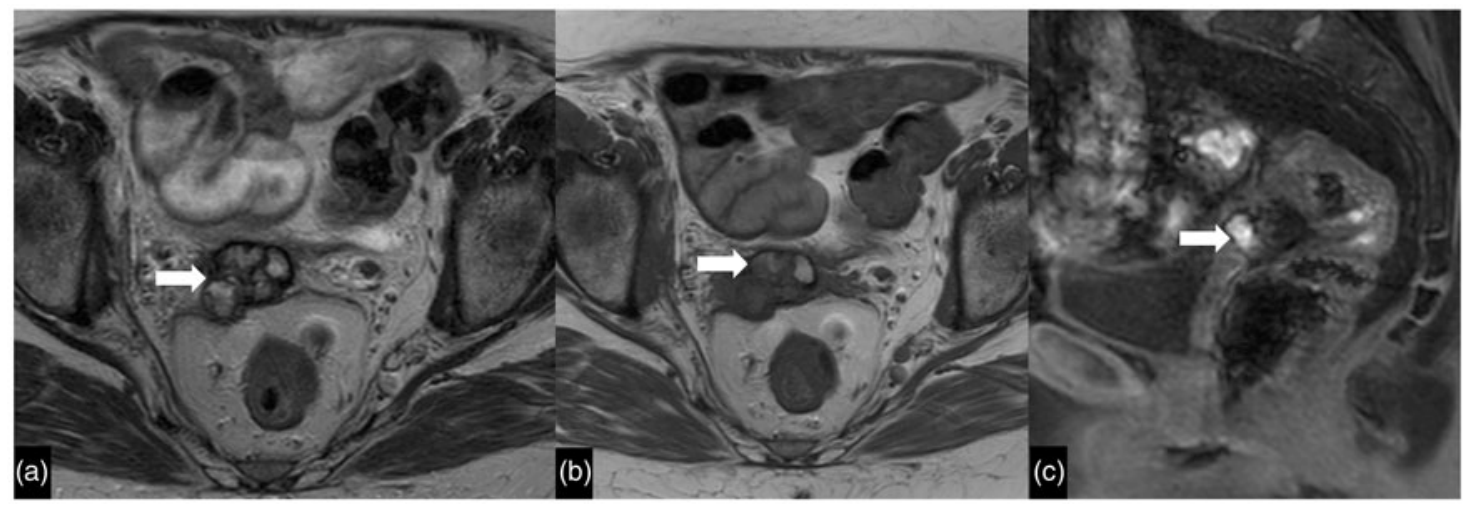

Figure 4. Adenocarcinoma with high signal intensity foci on T1-weighted images. Axial T2 weighted image (a), axial T1 weighted image (b) and sagittal T1 FS weighted imaging of an adenocarcinoma. We can see that it shows high signal intensity foci on $\mathrm{T} 1$, with no shading on $\mathrm{T} 2$, probably due to haemorrhagic content. 
Table 2. Location of the tumours.

\begin{tabular}{|c|c|c|c|c|c|}
\hline & Squamous cell carcinoma & Adenocarcinoma & Glassy cell carcinoma & Melanoma & Botryoid sarcoma \\
\hline \multicolumn{6}{|l|}{ Location (third) } \\
\hline Upper third & $16(59.3 \%)$ & $4(80 \%)$ & & & \\
\hline Medium third & $1(3.7 \%)$ & & & & \\
\hline Lower third & $1(3.7 \%)$ & & & $1(100 \%)$ & \\
\hline Superior and medium thirds & $4(14.8 \%)$ & & $1(100 \%)$ & & \\
\hline Medium and inferior thirds & $1(3.7 \%)$ & & & & \\
\hline All thirds & $4(14.8 \%)$ & $1(20 \%)$ & & & $1(100 \%)$ \\
\hline Total & 27 & 5 & 1 & 1 & 1 \\
\hline \multicolumn{6}{|l|}{ Location (wall) } \\
\hline Anterior & & $4(80 \%)$ & & $1(100 \%)$ & $1(100 \%)$ \\
\hline Posterior & $15(62.5 \%)$ & & $1(100 \%)$ & & \\
\hline Right & $4(16.7 \%)$ & $1(20 \%)$ & & & \\
\hline Left & $4(16.7 \%)$ & & & & \\
\hline Circumferential & $1(4.2 \%)$ & & & & \\
\hline Total & 24 & 5 & 1 & 1 & 1 \\
\hline
\end{tabular}

Table 3. FIGO staging.

\begin{tabular}{|c|c|c|c|c|c|}
\hline \multirow[b]{2}{*}{ Histologic type } & \multicolumn{5}{|c|}{ FIGO } \\
\hline & I & ॥ & III & IVa & $\mathrm{IVb}$ \\
\hline Squamous cell carcinoma & $13(48.1 \%)$ & $11(40.7 \%)$ & $1(3.7 \%)$ & $2(7.4 \%)$ & \\
\hline Adenocarcinoma & $2(40 \%)$ & $3(60 \%)$ & & & \\
\hline
\end{tabular}

Table 4. History of previous hysterectomy.

\begin{tabular}{|c|c|c|c|c|c|c|}
\hline \multirow[b]{4}{*}{ Histologic type } & \multicolumn{6}{|c|}{ Previous hysterectomy } \\
\hline & \multirow[b]{3}{*}{ No } & \multicolumn{5}{|c|}{ Yes } \\
\hline & & & \multicolumn{3}{|c|}{ Cervical Dysplasia } & \multirow[b]{2}{*}{ Endometrial cancer } \\
\hline & & Benign pathology & $\mathrm{CIN}^{\mathrm{a}} \mathrm{I}$ & $\mathrm{CIN}^{\mathrm{a}} \|$ & $\mathrm{CIN}^{\mathrm{a}} \mathrm{III}$ & \\
\hline Squamous cell carcinoma & 12 & 3 & 1 & 1 & 9 & 1 \\
\hline Adenocarcinoma & 1 & 4 & & & & \\
\hline Total & 13 & 19 & & & & \\
\hline
\end{tabular}

${ }^{\mathrm{a}}$ Cervical intraepithelial neoplasia (CIN).

in endometrial implants, which show high signal intensity on T1-weighted imaging and shading on T2-weighted images (Siegelman et al. 1997). This fact could explain the presence of high signal intensity foci on adenocarcinoma, although in our case shading was absent. There were also no other features of endometriosis in the pelvis. Because of that, we think that those foci may correspond to intralesional haemorrhage.

Our case of melanoma did not show high signal intensity on T1 unlike what was expected (Moon et al. 1993; Parikh et al. 2008; Grant et al. 2010) because of low melanin content that was histologically proven. Regarding location, we found that both SCC (59.3\%) and adenocarcinomas (80\%) were predominantly confined to the upper third of vagina, as was already shown in previous studies (Parikh et al. 2008; Grant et al. 2010). When analysing the wall of origin, the majority of SCCs originated on the posterior wall (62.5\%) and adenocarcinomas originated mainly on the anterior wall, with statistical significance. These data have also been already mentioned in previous studies (Parikh et al. 2008; Grant et al. 2010).

Differently from a previous study conducted by Dixit et al. (1993), where it was found that only $25.7 \%$ of SCCs presented at an early stage (I or II), in our study $88.9 \%$ of SCCS were FIGO I or II. This difference can be related to an improvement in surveillance and medical care over the years, allowing earlier diagnoses.
Although current treatment guidelines of vaginal cancer are based on the FIGO stage, regardless of the histologic type, there is still much discussion and controversy since this is a rare entity and many guidelines are extrapolated from experience and outcomes in cervical cancer (Gardner et al. 2015; Tsili 2019). Even so, histologic type and primary tumour characteristics are also predictors of survival. In fact, for females with SCC the 5-year survival is around 54\%; for adenocarcinoma is $\sim 60 \%$, while for melanoma is $13 \%$ (Gardner et al. 2015). Another important difference among histologic types is the recurrence rate since, for example, adenocarcinomas are more likely to recur than SCCs within 5 years after the diagnosis (Tsili 2019). Because of all of these factors, the histologic type has also clinical and management impact.

When analysing previous hysterectomies, we found a high number in both groups (56\% for SCCs group and $80 \%$ in the adenocarcinomas group). However, in the adenocarcinomas group, all hysterectomies were performed because of benign pathology, while in the SCCs group there was an association with cervical dysplasia (73\%). This association can be explained by the fact that risk factors for the development of vaginal cancer are similar to those of cervical cancer, including chronic HPV infection, multiple sexual partners, immunosuppression,and smoking (Grant et al. 2010). We highlight the role of HPV infection since it is strongly associated with the precursor of vaginal carcinoma and vaginal intraepithelial 
neoplasia. It has been proven that an increased incidence of vaginal carcinoma is observed in women with a previous diagnosis of cervical cancer or cervical intraepithelial neoplasia (López et al. 2005; Parikh et al. 2008; Grant et al. 2010; Walker et al. 2011). In fact, a previous study (Liu et al. 2013) points out that women over 60 years of age with a history of CIN lesions have an elevated risk of vaginal cancer and should be followed closely.

A study from Gante et al. (2017), which reviews the data from hysterectomies in Portugal between 2000 and 2014, shows a rate of hysterectomies between $212 / 100,000$ to $171 /$ 100,000 women per year. In that study, it is shown that benign pathology and uterine prolapse accounted for the majority of hysterectomies in Portugal in the last years (70\%). These data hint at the possibility that our high proportion of cases of cervical dysplasia in the SCCs group is related to the development of vaginal cancer.

The high rate of hysterectomies performed for benign disease may explain why we have such a high rate of previous hysterectomies in the adenocarcinoma group since the sample is small. Besides that, as we already pointed out, the diagnosis of primary vaginal malignancy implies that the tumour arises exclusively from the vagina, with no superior involvement of the external os (Parikh et al. 2008). This fact may also explain the higher incidence of vaginal cancer in women with previous hysterectomy even for benign disease since the cervix is absent (Taylor et al. 2007). We postulate that if these women did not have a previous hysterectomy, the tumour could also involve the cervix and so be classified as cervical cancer.

The main limitation is its retrospective design. For clinical aspects, we had to rely on the registrations present in the clinical charts. Regarding MRI examinations, since many of them were performed at other institutions previous to the admission of the patient in our hospital, we could not use a standard protocol and because of that some important sequences were missing in many of the patients, like dynamic contrast-enhanced imaging and diffusion-weighted imaging. Therefore, our study was mostly focussed on the T2 and T1-signal analysis.

Another important limitation is the small sample size, particularly in the adenocarcinomas group, which is related to its rarity. However, we think that this study may be a starting point for further investigation on the MRI features of these rare tumours.

\section{Conclusions}

Adenocarcinomas may show heterogeneous T2-signal, with high-signal intensity areas (probably corresponding to mucin components). On the other hand, SCCs are usually homogeneous on T2WI, unless they are poorly differentiated. Both SCC and adenocarcinomas usually involve the upper third of the vagina. However, adenocarcinomas most often arise from the anterior vaginal wall, while SCCs tend to arise from the posterior vaginal wall.

There is an association between vaginal SCC and a previous hysterectomy with cervical dysplasia, probably because they share many risk factors, including chronic HPV infection.

\section{Disclosure statement}

No potential conflict of interest was reported by the authors.

\section{References}

Chang YCF, Hricak H, Thurnher S, Lacey CG. 1988. Vagina: evaluation with MR imaging. Part II. Neoplasms. Radiology 169:175-179.

Dixit S, Singhal S, Baboo HA. 1993. Squamous cell carcinoma of the vagina: a review of 70 cases. Gynecologic Oncology 48:80-87.

Fetissof F, Haillot O, Lanson Y, Arbeille B, Lansac J. 1990. Papillary tumour of the vagina resembling transitional cell carcinoma. Pathology, Research and Practice 186:358-364.

Gante I, Medeiros-Borges C, Águas F. 2017. Hysterectomies in Portugal (2000-2014): what has changed? European Journal of Obstetrics, Gynecology, and Reproductive Biology 208:97-102.

Gardner CS, Sunil J, Klopp AH, Devine CE, Sagebiel T, Viswanathan C, Bhosale PR. 2015. Primary vaginal cancer: role of MRI in diagnosis, staging and treatment. The British Journal of Radiology 88:20150033

Grant LA, Sala E, Griffin N. 2010. Congenital and acquired conditions of the vulva and vagina on magnetic resonance imaging: a pictorial review. Semin Ultrasound CT MR 31:347-362.

Liu X, Yue Y, Zong S. 2013. Post-hysterectomy vaginal cuff cancer secondary to HPV infection and CIN: A case report. Pakistan Journal of Medical Sciences 29:1068-1070.

López C, Balogun M, Ganesan R, Olliff JF. 2005. MRI of vaginal conditions. Clinical Radiology 60:648-662.

Moon WK, Kim SH, Han MC. 1993. MR Findings of malignant melanoma of the vagina. Clinical Radiology 48:326-328.

Parikh JH, Barton DPJ, Ind TEJ, Sohaib SA. 2008. MR imaging features of vaginal malignancies. Radiographics 28:49-63.

Randall ME, Andersen WA, Mills SE, Kim JA. 1986. Papillary squamous cell carcinoma of the uterine cervix: a clinicopathologic study of nine cases. International Journal of Gynecological Pathology 5:1-10.

Siegelman ES, Outwater EK, Banner MP, Ramchandani P, Anderson TL, Schnall MD. 1997. High-resolution MR imaging of the vagina. Radiographics 17:1183-1203.

Taylor MB, Dugar N, Davidson SE, Carrington BM. 2007. Magnetic resonance imaging of primary vaginal carcinoma. Clinical Radiology 62: 549-555.

Tsili AC. 2019. Vagina and vulva. In: Forstner R, Cunha TM, Hamm B, editors. MRI and CT of the Female Pelvis. Berlin, Heidelberg: Springer. p. 343-368.

Walker DK, Salibian RA, Salibian AD, Belen KM, Palmer SL. 2011. Overlooked diseases of the vagina: a directed anatomic-pathologic approach for imaging assessment. Radiographics 31:1583-1598. 